\title{
Structural and Strongly Structural Input and State Observability of Linear Network Systems
}

\author{
Sebin Gracy, Federica Garin and Alain Y. Kibangou.
}

\begin{abstract}
This paper studies linear network systems affected by multiple unknown inputs with the objective of reconstructing both the initial state and the unknown input with one timestep delay. We state conditions under which both the whole network state and the unknown input can be reconstructed from output measurements, over every window of length $N, N$ being the dimension of the system, for all system matrices that share a common zero/non-zero pattern (uniform $N$-step strongly structural input and state observability) or at least for almost all system matrices that share a common zero/non-zero pattern (uniform $N$-step structural input and state observability). Based on some specific assumptions on the structure of the interactions between the unknown input and the network states, we show that such a characterization depends only on strongly structural (resp. structural) observability properties of a suitable subsystem.
\end{abstract}

Index Terms-Linear Network systems, Input and State Observability (ISO), Structural Observability, Strongly Structural Observability, Cyber-Physical Security.

\section{INTRODUCTION}

The fields of application of network systems span from critical infrastructure domains such as power networks, water and gas distribution networks to healthcare systems, flight control systems among others. Given the ubiquitous nature of their usage, it is of paramount importance to ensure that each individual subsystem functions as desired. The notion of observability enables one to achieve this by expending as few resources as possible.

However, such systems are quite likely to malfunction due to local attacks by malicious agents modeled as external unknown inputs [1] which could have significant consequences as evidenced by the failure of wastewater management systems in Marochy Australia in early 2000 [2], multiple power blackouts in Brazil [3], to cite a few. Hence, in addition to being able to observe the state in the presence of unknown inputs (also known as strong observability [4], [5]) it is crucial that the unknown input be observed as well. This notion is known as Input and State Observability, which hereafter is referred to as ISO. In the context of Linear Time Varying (LTV) systems, a system being ISO over an interval does not necessarily mean that the said system would be ISO over every sufficiently long interval. The concept of uniform $\delta$-step ISO (i.e., ISO over every time window of length $\delta$ ) gets rid of this drawback. The notion of ISO is of particular importance in designing unbiased minimum-variance filters that simultaneously estimate both state and unknown input [6]-[8]. It is well-known

The authors are with Univ. Grenoble Alpes, CNRS, Inria, Grenoble INP, GIPSA-Lab, F-38000, Grenoble, France. (e-mail: sebin.gracy@inria.fr, federica.garin@inria.fr, alain.kibangou@univ-grenoble-alpes.fr). that algebraic approaches towards characterizing ISO involve the classic Kalman-like rank condition or a variant of the Popov-Belevitch-Hautus (PBH) test (see e.g. [9], [8]). Both tests require exact knowledge of entries in the matrices of interest and are computationally heavy as the dimension of the system grows, while the latter is not suitable for LTV systems. This leads to the study of ISO based on the structure of the underlying network (represented by a graph) and the corresponding line of work is known as structured systems.

We say that a linear system is structured if the system matrices have coefficients that are either a fixed zero or a free parameter (i.e., the coefficients may take any value in $\mathbb{R}$ ). Thus, one can capture a family of systems that obey a certain rule. Under such a setup, if a property holds for almost all choices of entries in the non-zero positions of the system matrices it is called structural [10], whereas if it holds for all non-zero choices of entries in the non-zero positions of the system matrices it is called strongly structural [11] (s-structural).

For linear time-invariant (LTI) systems, structural controllability or the dual notion of observability has been studied since [10] while graph-theoretic characterizations for s-structural controllability were first provided in [11]. The survey paper [12] revises some graph-theoretic characterizations for observability in addition to recalling similar conditions for solvability issues like disturbance rejection, input-output decoupling, and so on, while equivalent characterizations for s-structural controllability have been provided in [13]. More recently, for sstructural controllability, [14] provides necessary and sufficient conditions in terms of uniquely restricted matching (precise definition appears in Section VI) while [15] does so in terms of zero forcing sets.

Notice that all the results mentioned as yet are for LTI systems, where both parameters and the structure remain constant over the time. In contrast, it is natural to assume that the parameters can evolve over the time while the structure remains fixed (LTV with fixed topology). Under such a scenario, necessary and sufficient conditions for structural observability of $L T V$ systems are given in [16] while necessary and sufficient conditions for s-structural observability are available in [17]. However, these results are not applicable for LTV systems with unknown inputs.

To the best of our knowledge, for discrete-time linear structured systems, a graph-theoretic characterization for the more general ISO problem encompassing multiple unknown inputs and accounting for $L T V$ dynamics, is missing. For continuoustime LTI systems, [18] gives necessary and sufficient conditions for structural ISO, which when translated into a discretetime setup yields structural ISO with some delay $L$. The 
present paper deals with the notion of ISO with delay-1, a notion that is essential for running input and state estimation filters. For discrete-time LTV systems, [19] gives a characterization of s-structural ISO but not of structural ISO while an earlier work from the authors gives characterizations of both structural and s-structural ISO for LTI systems affected by a single unknown input [20]. As such the main contributions of this paper are threefold; under suitable assumptions on the structure of the input and output matrices, first we show equivalence between ISO of a linear system and observability of a suitably defined subsystem. Second, we give a characterization of uniform $N$-step structural (see Theorem 1) (resp. uniform $N$-step strongly structural (see Theorem 2)) input and state observability, i.e., the conditions under which both the whole network state and the unknown input can be reconstructed for almost all (resp. all) system matrices that share a common zero/non-zero pattern, over every time window of length $N$. This equivalence enables one to study structural (resp. sstructural) ISO using the graph techniques given in [21], [14] (resp. [14], [15]).

The organization of this paper is as follows: We state the problem in Section II. Section III gives an algebraic characterization of the ISO problem while Section IV, under suitable assumptions on the input and output matrices, shows the equivalence between ISO and observability of an appropriate subsystem. Section V discusses structural ISO while Section VI studies the stronger notion of s-structural ISO. Differently from Section V and VI, Section VII explores structural and s-structural ISO without assumptions on input and output matrices, while Section VIII gives concluding remarks along with discussing future lines of research.

\section{Notations}

$\mathbb{R}, \mathbb{R}_{*}$ and $\mathbb{Z}$ denote the set of real numbers, non-zero real numbers and integers respectively. $e_{j ; N}$ represents the $j^{t h}$ vector of the canonical basis of $\mathbb{R}^{N}$. Alternatively, assuming that the length is clear from context, we would denote the same as just $e_{j} .[A]_{i, j}$ denotes the entry in matrix $A$ that corresponds to its $i^{\text {th }}$ row and $j^{\text {th }}$ column. $I_{N}$ denotes an identity matrix of size $N$. Given two matrices $A$ and $B$, let $A \odot B$ and $A \otimes B$ denote the entrywise product and Kronecker product respectively. $A=\operatorname{diag}\left(A_{1}, A_{2}, \ldots, A_{N}\right)$ denotes a block diagonal matrix whose blocks along the diagonal are $A_{1}, A_{2}, \ldots, A_{n}$. In case $A_{1}=A_{2}=\ldots=A_{N}$, we get $\operatorname{diag}\left(A_{1}, A_{2}, \ldots, A_{N}\right)=I_{N} \otimes A .\left\{A_{k}\right\}_{k_{0}}^{k_{1}}$ denotes a sequence of matrices $A_{k}, k=k_{0}, k_{0}+1, \cdots, k_{1} \cdot|\mathcal{X}|$ denotes cardinality of a set $\mathcal{X}$. $\lceil a\rceil$ denotes the smallest integer greater than or equal to $a$.

\section{Problem Statement}

Consider a linear network system with $N$ nodes, represented by a graph $\mathcal{G}=\{\mathcal{V}, \mathcal{E}\}$ where $\mathcal{V}$ is the vertex set and $\mathcal{E}=$ $\left\{(j, i) \in \mathcal{V} \times \mathcal{V} \mid\left[A_{\mathcal{G}}\right]_{i, j}=1\right\} ; A_{\mathcal{G}}$ being the adjacency matrix of $\mathcal{G}$. In this network, some states can be directly measured. They define the set $\mathcal{O}=\left\{j_{1}, j_{2}, \cdots, j_{M}\right\} \subseteq \mathcal{V}, M$ being the number of observed states. From an analysis of the network, we assume that $\mathcal{V}$ can also be partitioned into assailable nodes and reliable ones. We define by $\mathcal{A}=\left\{i_{1}, i_{2}, \cdots, i_{R}\right\}$ the set of the $R$ assailable nodes that may be attacked by $P$ external malicious agents defining the set denoted by $\mathcal{I}$, the attack being modeled as a unknown input. An illustration is given in Fig. 1 where three malicious nodes, namely, $x, y, z$ can attack the network with vertex set $\mathcal{V}$ through agents $k, j$ and $i$. A setup of this sort can be used as an abstraction to model attacks on multiple nodes including deception attacks [22], false data injection [23], fault diagnosis and detection [24], input estimation in physiological systems [25].

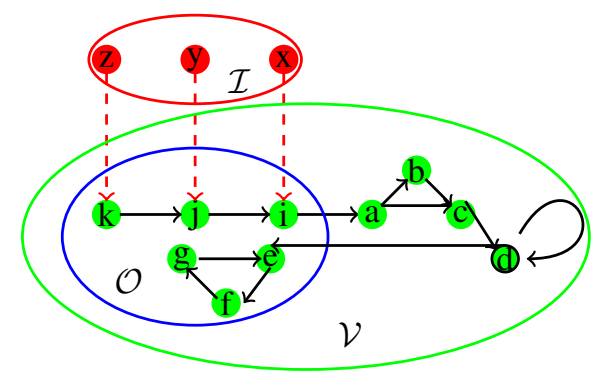

Fig. 1: Graph representation of a network system affected by external agents where $\mathcal{I}, \mathcal{O}$ and $\mathcal{V}$ are the sets of attackers, observed nodes and state nodes respectively, while the set of assailable nodes is $\mathcal{A}=\{i, j, k\}$.

The dynamics of the linear network system described above is given by the following equations:

$$
\begin{aligned}
x_{k+1} & =W_{k} x_{k}+A_{B} u_{k} \\
y_{k} & =A_{C} x_{k}
\end{aligned}
$$

with state vector $x_{k} \in \mathbb{R}^{N}$, unknown input vector $u_{k} \in \mathbb{R}^{P}$ and output vector $y_{k} \in \mathbb{R}^{M}$. Furthermore, $W_{k} \in \mathbb{R}^{N \times N}$, $A_{B} \in \mathbb{R}^{N \times P}$ and $A_{C} \in \mathbb{R}^{M \times N}$, where $W_{k}, A_{B}$ and $A_{C}$ are state matrix at time instant $\mathrm{k}$, input matrix and output matrix respectively. For the rest of this paper, over a given interval $\left[k_{0}, k_{1}\right]$, we denote the dynamics of a LTV system as $\left\{W_{k}, A_{B}, A_{C}\right\}_{k_{0}}^{k_{1}}$ while that of a LTI system is indicated by $\left\{W, A_{B}, A_{C}\right\}_{k_{0}}^{k_{1}}$.

In what follows we assume that for all $k$ belonging to $\mathbb{Z}$, $W_{k} \in \mathcal{W}$ or in particular $W_{k} \in \mathcal{W}^{*}$, where matrix set $\mathcal{W}=\left\{Z_{1} \odot A_{\mathcal{G}} \mid Z_{1} \in \mathbb{R}^{N \times N}\right\}$ and $\mathcal{W}^{*}=\left\{Z_{1} \odot A_{\mathcal{G}} \mid\right.$ $\left.Z_{1} \in \mathbb{R}_{*}^{N \times N}\right\}$. Both $\left\{\mathcal{W}, A_{B}, A_{C}\right\}$ and $\left\{\mathcal{W}^{*}, A_{B}, A_{C}\right\}$ impose a fixed zero structure. The remaining coefficients (i.e., not fixed to zero positions) of the matrices are referred to as free parameters. The free parameters of matrices belonging to $\mathcal{W}$ can take any values while those belonging to $\mathcal{W}^{*}$ strictly take non-zero values. Notice that this assumption implies that the topology of $\mathcal{G}$ remains fixed but the entries corresponding to the free parameters of the system matrices may vary. For the particular case of LTI systems, the said entries remain constant.

We narrow our attention to the case wherein each unknown input affects exactly one node of $\mathcal{G}$ and each node is at most affected by a single unknown input. This leads to the following assumption:

Assumption 1 (Al):

$$
A_{B}=\left[\begin{array}{lllll}
e_{i_{1} ; N} & e_{i_{2} ; N} & \cdot & e_{i_{P} ; N}
\end{array}\right],
$$




$$
A_{C}^{T}=\left[\begin{array}{lllll}
e_{j_{1} ; N} & e_{j_{2} ; N} & \cdot & \cdot & e_{j_{M} ; N}
\end{array}\right]
$$

In the context of network systems, it is natural to think of states as local variables that are in different physical locations, whereas unknown inputs could be isolated entities that are at best able to attack a single state. For instance, the topology of a power distribution network can be considered as the connectivity between the meters installed at the substation, feeders, transformers, and consumer mains. An attack corresponds to addition or draining of active power while the state at each node can be measured using smart meters.

As a consequence of assumption $\mathrm{A} 1$, we rule out scenarios wherein a linear combination of multiple unknown inputs affects a single node in $\mathcal{G}$. Therefore, we have $R=P$. On the other hand, the unknown inputs are of arbitrary nature and for the particular case in which some of the unknown inputs are the same, we would have a single unknown input affecting multiple nodes in $\mathcal{G}$ and as such we provide sufficient but not necessary conditions for this setup as well.

In this paper, we first study conditions under which it is possible to jointly estimate both the initial state and the sequence of multiple unknown inputs for an LTV system $\left\{W_{k}, A_{B}, A_{C}\right\}_{k_{0}}^{k_{1}}$ as well as the particular case of an LTI system $\left\{W, A_{B}, A_{C}\right\}_{k_{0}}^{k_{1}}$ from measurements of a subset of state vertices. Thereafter, based only on the structure of the graph $\mathcal{G}$, we will characterize ISO for i) almost all choices of entries in $\mathcal{W}$ (see Section $V$ ) and ii) every choice of entries in $\mathcal{W}^{*}$ (see Section VI), over all sufficiently long time windows.

\section{Algebraic CharacterizATION}

In this section we describe some algebraic criteria for observability and for ISO, reviewing the relevant classical results together with some new variation of them concerning ISO, see in particular Prop. 4.

\section{A. Definitions}

The concept of observability was first introduced by Kalman in his seminal paper [26]. We recall it in the following definition.

Definition 1: The system $\left\{W_{k}, A_{C}\right\}_{k_{0}}^{k_{1}}$ is observable on $\left[k_{0}, k_{1}\right]$ if any initial state $x_{k_{0}}$ is uniquely determined by the corresponding measured output sequence $\left\{y_{k_{0}}, y_{k_{0}+1}, \ldots, y_{k_{1}}\right\}$.

It is worthwhile to notice here that Definition 1 explicitly asks that the initial state $x_{k_{0}}$ be reconstructed, assuming that the input is known. On the other hand, the notion of strong observability asks that the initial state $x_{k_{0}}$ be reconstructed even in the presence of an unknown input while that of left invertibility with delay 1 requires that the inputs from $u_{k_{0}}$ up to $u_{k_{1}-1}$ be reconstructed from the outputs up to $y_{k_{1}}$. These two notions, namely, strong observability and left invertibility with delay 1 , give rise to the definition of $I S O$.

Definition 2: The system $\left\{W_{k}, A_{B}, A_{C}\right\}_{k_{0}}^{k_{1}}$ is ISO, with delay 1 , on the interval $\left[k_{0}, k_{1}\right]$ if the initial condition $x_{k_{0}} \in \mathbb{R}^{N}$ and the unknown inputs sequence $\left\{u_{k_{0}}, u_{k_{0}+1}, \ldots, u_{k_{1}-1}\right\}$ can be uniquely determined from the measured output sequence $\left\{y_{k_{0}}, y_{k_{0}+1}, \ldots, y_{k_{1}}\right\}$.
A stronger notion of observability is that of uniform $\delta$-step observability which requires that a system be observable over every time window of length $\delta$ [27]. Analogously, we define uniform $\delta$-step ISO as follows:

Definition 3: The system $\left\{W_{k}, A_{B}, A_{C}\right\}_{k \in \mathbb{Z}}$ is uniformly $\delta$ step ISO if $\forall k_{0} \in \mathbb{Z}\left\{W_{k}, A_{B}, A_{C}\right\}_{k_{0}}^{k_{0}+\delta}$ is ISO over $\left[k_{0}, k_{0}+\right.$ $\delta]$.

Remark 1: Notice that although uniform $\delta$-step ISO (resp. observability) is with respect to all intervals of length $\delta$, it turns out that it can be rephrased considering all intervals of length at least $\delta$. For observability, this is immediate: if a system is observable over $\left[k_{0}, k_{0}+\delta\right]$, then it is also observable over $\left[k_{0}, k_{0}+\eta\right]$ for all $\eta \geq \delta$. For ISO, one needs to reconstruct all inputs up to $k_{0}+\eta-1$ and not only those up to $k_{0}+\delta-1$. If the system is uniformly $\delta$-step observable, it is possible to use $\delta$-step ISO over successive time windows of length $\delta$ to ensure that all the required inputs are indeed reconstructed.

Remark 2: It is well-known that either LTI systems are not observable or are uniformly $N$-step observable in which case we would simply call it as observable.

\section{B. Observability, Invertibility, Input and State Observability Matrices}

Let $\Theta_{k_{0}, k_{1}}, \Gamma_{k_{0}, k_{1}}$ and $\Psi_{k_{0}, k_{1}}$ represent the observability matrix, invertibility matrix, and input and state observability (ISO) matrix respectively over the interval $\left[k_{0}, k_{1}\right]$. These are defined as follows:

$$
\begin{aligned}
& \Theta_{k_{0}, k_{1}}=\left[\begin{array}{c}
A_{C} \\
A_{C} W_{k_{0}} \\
A_{C} W_{k_{0}+1} W_{k_{0}} \\
\cdot \\
\cdot \\
\cdot \\
A_{C} W_{k_{1}-1} \cdots W_{k_{0}}
\end{array}\right] \\
& \Gamma_{k_{0}, k_{1}}=\left[\begin{array}{cccc}
0 & \ldots & \ldots & 0 \\
A_{C} A_{B} & \ldots & \ldots & 0 \\
A_{C} W_{k_{0}+1} A_{B} & A_{C} A_{B} & \ldots & 0 \\
\cdot & \cdot & \ldots & \cdot \\
\cdot & \cdot & \ldots & \cdot \\
A_{C} W_{k_{1}-1} \cdots W_{k_{0}+1} A_{B} & \ldots & \ldots & A_{C} A_{B}
\end{array}\right], \\
& \Psi_{k_{0}, k_{1}}=\left[\Theta_{k_{0}, k_{1}} \Gamma_{k_{0}, k_{1}}\right] .
\end{aligned}
$$

This leads us to the following classical Kalman-like characterization of ISO.

Proposition 1: The system $\left\{W_{k}, A_{B}, A_{C}\right\}_{k_{0}}^{k_{1}}$ is ISO over $\left[k_{0}, k_{1}\right]$ if and only if $\Psi_{k_{0}, k_{1}}$ is full column rank, i.e. $\operatorname{rank}\left(\Psi_{k_{0}, k_{1}}\right)=N+\left(k_{1}-k_{0}\right) P$.

Proof: Let $y_{k_{0}: k_{1}}$ and $u_{k_{0}: k_{1}-1}$ denote the vectors of concatenated outputs and unknown inputs over $\left[k_{0}, k_{1}\right]$, respectively. Therefore, from (1) and (2) the following can be readily obtained

$y_{k_{0}: k_{1}}=\Theta_{k_{0}, k_{1}} x_{k_{0}}+\Gamma_{k_{0}, k_{1}} u_{k_{0}: k_{1}-1}=\Psi_{k_{0}, k_{1}}\left[\begin{array}{c}x_{k_{0}} \\ u_{k_{0}: k_{1}-1}\end{array}\right]$. 
Based on Definition 2 it is immediate that input and state observability is equivalent to uniqueness of the above system of linear equations.

Prop. 1 enables one to exploit the structure of $\Psi_{k_{0}, k_{1}}$ so as to find some simple necessary conditions for $\Psi_{k_{0}, k_{1}}$ to have full column rank. The following proposition briefly summarizes them.

Proposition 2: The following conditions are necessary for the system $\left\{W_{k}, A_{B}, A_{C}\right\}_{k_{0}}^{k_{1}}$ to be ISO over $\left[k_{0}, k_{1}\right]$ :

i) $\operatorname{rank}\left(\Theta_{k_{0}, k_{1}}\right)=N$,

ii) $\operatorname{rank}\left(A_{C} A_{B}\right)=P$,

iii) $M \geq P$.

iv) $N \geq P$

In case $N>P$, then the following conditions are also necessary:

v) $M>P$

vi) $k_{1}-k_{0} \geq\left[\frac{N-M}{M-P}\right]$.

In case $P=N$ then the following conditions are necessary and sufficient:

$M=N, \forall k \in\left[k_{0}, k_{1}\right] \operatorname{rank}\left(A_{C}\right)=N$ and $\forall k \in\left[k_{0}, k_{1}-1\right]$ $\operatorname{rank}\left(A_{B}\right)=N$.

Proof: The proof is reported in the Appendix. $\square$.

Notice that Prop. 2 fully characterizes the ISO problem for the particular case of $P=N$ where, under $\mathrm{A} 1$, the system is ISO if and only if all nodes are observed (i.e., $\mathcal{O}=\mathcal{V}$ ). In this paper we restrict our attention to the non-trivial case of $N>P$ i.e., not all the nodes are assailable. Therefore, from Prop. 2, $M>P$ is a necessary condition for ISO.

From Prop. 2 we know that the following are necessary conditions for ISO:

1) all the assailable nodes are observed i.e., $\left\{i_{1}, i_{2}, . ., i_{P}\right\} \subset$ $\left\{j_{1}, j_{2}, \ldots, j_{M}\right\}$ and

2) all of the assailable nodes are distinct i.e., there does not exist $h, k$ belonging to $\{1,2 \ldots, P\}$ such that $i_{h}=i_{k}$.

We also assume that all of the observed nodes are distinct i.e., there does not exist $h, k$ belonging to $\{1,2 \ldots, M\}$ such that $j_{h}=j_{k}$. This ensures that there are no repeated or dependent rows in $C$. Therefore, one can relabel the nodes in $\mathcal{G}$ in the following manner: $i_{1}=j_{1}=1, i_{2}=j_{2}=2, \ldots, i_{P}=j_{P}=P$. The aforesaid relabeling allows us to rewrite $A_{B}$ and $A_{C}$ as follows:

Assumption 2 (A2):

$$
\begin{aligned}
& A_{B}=\left[\begin{array}{llll}
e_{1 ; N} & e_{2 ; N} & \ldots & e_{P ; N}
\end{array}\right], \\
& A_{C}^{T}=\left[\begin{array}{lllllll}
e_{1 ; N} & e_{2 ; N} & \ldots & e_{P ; N} & e_{j_{P+1} ; N} & \ldots & e_{j_{M} ; N}
\end{array}\right]
\end{aligned}
$$

\section{Alternative Algebraic Characterization}

Prop. 1 characterizes ISO in terms of rank of $\Psi_{k_{0}, k_{1}}$. However, the elements in $\Psi_{k_{0}, k_{1}}$ are obtained by taking products of the state matrices over the interval $\left[k_{0}, k_{1}\right]$. Consequently, the zero/non-zero pattern is not preserved. In order to overcome this drawback, in this subsection we provide an alternative algebraic characterization for both observability and ISO.

Theorem 6.4.1 in [28] gives an alternative characterization of controllability. The following proposition does the same for observability.
Proposition 3: The system $\left\{W_{k}, A_{C}\right\}_{k_{0}}^{k_{1}}$ is observable over $\left[k_{0}, k_{1}\right]$ if and only if $\operatorname{rank}\left(\mathcal{Q}_{k_{0}, k_{1}}\right)=\left(k_{1}-k_{0}+1\right) N$, where

$$
\mathcal{Q}_{k_{0}, k_{1}}=\left[\begin{array}{ccccc}
A_{C} & 0 & \ldots & \ldots & 0 \\
0 & A_{C} & \ldots & \ldots & 0 \\
. & \ldots & \ldots & \ldots & . \\
. & \ldots & \ldots & \ldots & \cdot \\
0 & \ldots & \ldots & \ldots & A_{C} \\
W_{k_{0}} & -I_{N} & \ldots & \ldots & 0 \\
0 & W_{k_{0}+1} & -I_{N} & \ldots & 0 \\
. & \ldots & \ldots & \ldots & . \\
. & \ldots & \ldots & \ldots & . \\
0 & \ldots & \ldots & W_{k_{1}-1} & -I_{N}
\end{array}\right],
$$

with $\mathcal{Q}_{k_{0}, k_{1}} \in \mathbb{R}^{\left(k_{1}-k_{0}+1\right) M+\left(k_{1}-k_{0}\right) N \times\left(k_{1}-k_{0}+1\right) N}$. Proof: Notice that the problem of reconstructing $x_{k_{0}}$ from $y_{k_{0}: k_{1}}$ is equivalent to the problem of reconstructing $x_{k_{0}}, x_{k_{0}+1}, \ldots, x_{k_{1}}$. The relationship between the states and outputs can be expressed via a system of linear equations as follows. From Eq. (1) and setting $u(k)=\mathbf{0}_{P}$, we have: $\forall k \in\left[k_{0}, k_{1}-1\right], W_{k} x_{k}-x_{k+1}=\mathbf{0}_{N}$ and $\forall k \in\left[k_{0}, k_{1}\right]$ $C_{k} x_{k}=y_{k}$.

This can be rewritten as: $\mathcal{Q}_{k_{0}, k_{1}} x_{k_{0}: k_{1}}=\left[\begin{array}{c}y_{k_{0}: k_{1}} \\ \mathbf{0}_{\left(k_{1}-k_{0}-1\right) N}\end{array}\right]$. Hence, the system $\left\{W_{k}, A_{B}, A_{C}\right\}_{k_{0}}^{k_{1}}$ is observable, over $\left[k_{0}, k_{1}\right]$, if and only if the above system of linear equations has a unique solution.

It turns out that similar arguments can be made for ISO as well, and will be shown in Prop. 4. As a first step we define the following matrix:

$$
\mathcal{J}_{k_{0}, k_{1}}=\left[\begin{array}{cc}
0 & \mathcal{Q}_{k_{0}, k_{1}}
\end{array}\right],
$$

where $\mathbf{B}_{k_{0}, k_{1}}=I_{k_{1}-k_{0}} \otimes A_{B} \in \mathbb{R}^{\left(k_{1}-k_{0}\right) N \times\left(k_{1}-k_{0}\right) P}$.

Proposition 4: The system $\left\{W_{k}, A_{B}, A_{C}\right\}_{k_{0}}^{k_{1}}$ is ISO over $\left[k_{0}, k_{1}\right]$ if and only if $\operatorname{rank}\left(\mathcal{J}_{k_{0}, k_{1}}\right)=\left(k_{1}-k_{0}\right) P+\left(k_{1}-\right.$ $\left.k_{0}+1\right) N$.

Proof: The problem of reconstructing $x_{k_{0}}$ and $u_{k_{0}: k_{1}-1}$ from $y_{k_{0}: k_{1}}$ is equivalent to the problem of reconstructing $x_{k_{0}}, x_{k_{0}+1}, \ldots, x_{k_{1}}$ and $u_{k_{0}: k_{1}-1}$. From (1), we have: $\forall k \in\left[k_{0}, k_{1}-1\right], W_{k} x_{k}+A_{B} u_{k}-x_{k+1}=\mathbf{0}_{N}$ and $\forall k \in\left[k_{0}, k_{1}\right] A_{C} x_{k}=y_{k}$. Hence, both the state equation and output equation at each time instant can be expressed as a linear combination of $x_{k_{0}}, x_{k_{0}+1}, \ldots, x_{k_{1}}$ as well as $u_{k_{0}}, u_{k_{0}+1}, \ldots, u_{k_{1}-1}$, in the following manner:

$$
\mathcal{J}_{k_{0}, k_{1}}\left[\begin{array}{c}
u_{k_{0}: k_{1}-1} \\
x_{k_{0}: k_{1}}
\end{array}\right]=\left[\begin{array}{c}
y_{k_{0}: k_{1}} \\
\mathbf{0}_{\left(k_{1}-k_{0}\right) N}
\end{array}\right],
$$

Hence, the system $\left\{W_{k}, A_{B}, A_{C}\right\}_{k_{0}}^{k_{1}}$ is ISO over $\left[k_{0}, k_{1}\right]$ if and only if the above system of linear equations has a unique solution.

\section{ISO AS OBSERVABILITY OF AN APPROPRIATE SUBSYSTEM}

The objective here is to decompose the system $\left\{W_{k}, A_{B}, A_{C}\right\}_{k_{0}}^{k_{1}}$ into two subsystems and show that ISO is equivalent to observability of one of the subsystems. It is crucial to notice here that the identity of the nodes being 
assailable remains fixed and, according to assumption A2, equal to $\{1,2, \ldots, P\}$. Consequently, the nodes labeled from $i_{P+1}, \ldots, i_{N}$ are not assailable. This enables us to decompose the state vector in two blocks: $\hat{x}_{k}$ denoting states that are directly affected by the unknown inputs and $\tilde{x}_{k}$ for the remaining states; a corresponding partitioning is also done for the output vector, obtaining

$$
x_{k}=\left[\begin{array}{l}
\hat{x}_{k} \\
\tilde{x}_{k}
\end{array}\right], y_{k}=\left[\begin{array}{l}
\hat{y}_{k} \\
\tilde{y}_{k}
\end{array}\right],
$$

with $\hat{x}_{k} \in \mathbb{R}^{\mathrm{P}}, \tilde{x}_{k} \in \mathbb{R}^{\mathrm{N}-\mathrm{P}}, \hat{y}_{k} \in \mathbb{R}^{\mathrm{P}}$, and $\tilde{y}_{k} \in \mathbb{R}^{\mathrm{M}-\mathrm{P}}$. Moreover, thanks to Assumption $\mathrm{A} 2$, the input and output matrices can be rewritten as follows:

$$
A_{B}=\left[\begin{array}{c}
I_{P} \\
\mathbf{0}
\end{array}\right], A_{C}=\left[\begin{array}{cc}
I_{P} & \mathbf{0} \\
\mathbf{0} & \tilde{A}_{C}
\end{array}\right] \text {. }
$$

Therefore, the system $\left\{W_{k}, A_{B}, A_{C}\right\}_{k_{0}}^{k_{1}}$ can be decomposed into two subsystems as follows:

$$
\begin{gathered}
\left\{\begin{aligned}
\hat{x}_{k+1} & =\hat{W}_{k} \hat{x}_{k}+\Lambda \tilde{x}_{k}+u_{k} \\
\hat{y}_{k}= & \hat{x}_{k}
\end{aligned}\right. \\
\left\{\begin{array}{rll}
\tilde{x}_{k+1} & =\tilde{W}_{k} \tilde{x}_{k}+\Omega \hat{x}_{k} \\
\tilde{y}_{k} & =\tilde{A}_{C} \tilde{x}_{k}
\end{array}\right.
\end{gathered}
$$

where we use the notation

$$
W_{k}=\left[\begin{array}{cc}
\hat{W}_{k} & \Lambda_{k} \\
\Omega_{k} & \tilde{W}_{k}
\end{array}\right]
$$

From (3), it is clear that $\hat{x}_{k}$ is directly observed. Hence, (3) represents a system with known state but two unknown inputs, namely, $\tilde{x}_{k}$ and $u_{k}$, while (4) represents a system with unknown state but known input. Hence, we have the following proposition.

Proposition 5: Under A2, the system $\left\{W_{k}, A_{B}, A_{C}\right\}_{k_{0}}^{k_{1}}$ is ISO over $\left[k_{0}, k_{1}\right]$ if and only if the system $\left\{\tilde{W}_{k}, \tilde{A}_{C}\right\}_{k_{0}}^{k_{1}}$ is observable over $\left[k_{0}, k_{1}\right]$.

Proof: We define the matrices $Q_{N}$ and $\bar{Q}_{N}$ as follows:

$$
Q_{N}=\left[\begin{array}{c}
I_{p} \\
0
\end{array}\right], \bar{Q}_{N}=\left[\begin{array}{c}
0 \\
I_{N-P}
\end{array}\right] .
$$

Let $\Pi_{1}$ and $\Pi_{2}$ represent row and column permutation matrices respectively, defined as follows. For column permutations, we put at the beginning the first $P$ columns of each occurrence of $A_{C}$, obtaining

$$
\mathcal{J} \Pi_{2}=\left[\begin{array}{ccc}
R_{1} & 0 & R_{3} \\
R_{2} & \mathbf{B}_{k_{0}, k_{1}} & R_{4}
\end{array}\right],
$$

where $R_{1}=I_{k_{1}-k_{0}+1} \otimes A_{C} Q_{N}$,

$$
\begin{gathered}
R_{2}=\left[\begin{array}{ccccc}
W_{k_{0}} Q_{N} & -Q_{N} & 0_{N \times P} & \ldots & \ldots \\
\ldots & \ldots & \ldots & \ldots & \ldots \\
\ldots & \ldots & \ldots & \ldots & \ldots \\
0_{N \times P} & \ldots & \ldots & W_{k_{1}-1} Q_{N} & -Q_{N}
\end{array}\right], \\
R_{3}=I_{k_{1}-k_{0}+1} \otimes A_{C} \bar{Q}_{N}, \text { and } \\
R_{4}=\left[\begin{array}{cccc}
W_{k_{0}} \bar{Q}_{N} & -\bar{Q}_{N} & 0 & \ldots \\
\ldots & \ldots & \ldots & \ldots \\
\ldots & \ldots & \ldots & \ldots \\
0 & \ldots & W_{k_{1}-1} \bar{Q}_{N} & -\bar{Q}_{N}
\end{array}\right] .
\end{gathered}
$$

For row permutations, consider the following steps: we first arrange the $\left(k_{1}-k_{0}+1\right)$ row blocks corresponding to the first $P$ rows of each occurrence of $A_{C}$, then the $\left(k_{1}-k_{0}\right)$ row blocks corresponding to the first $P$ rows of each occurrence of $A_{B}$, and finally the remaining rows, so as to obtain

$$
\Pi_{1} \mathcal{J} \Pi_{2}=\left[\begin{array}{ccc}
\mathbf{I}_{\left(k_{1}-k_{0}+1\right) P} & 0 & 0 \\
P_{1} & \mathbf{I}_{\left(k_{1}-k_{0}\right) P} & P_{2} \\
0 & 0 & \tilde{\mathbf{C}} \\
P_{3} & 0 & \tilde{\mathbf{W}}
\end{array}\right],
$$

where

$$
\begin{gathered}
P_{1}=\left[\begin{array}{cccc}
\hat{W}_{k_{0}} & -I_{P} & 0 & \ldots \\
\ldots & \ddots & \ddots & \ldots \\
0 & \ldots & \hat{W}_{k_{1}-1} & -I_{P}
\end{array}\right], \\
P_{2}=\left[\begin{array}{cccc}
\Lambda_{k_{0}} & 0 & \ldots & \ldots \\
\ldots & \ddots & \ldots & \ldots \\
0 & \ldots & \Lambda_{k_{1}-1} & 0
\end{array}\right], \\
P_{3}=\left[\begin{array}{cccc}
\Omega_{k_{0}} & 0 & \ldots & \ldots \\
\ldots & \ddots & \ldots & \ldots \\
0 & \ldots & \Omega_{k_{1}-1} & 0
\end{array}\right],
\end{gathered}
$$

$\tilde{\mathbf{C}}=I_{k_{1}-k_{0}+1} \otimes A_{C}$, and

$$
\tilde{\mathbf{W}}=\left[\begin{array}{ccccc}
\tilde{W}_{k_{0}} & -I_{N-P} & \ldots & \ldots & 0 \\
0 & \tilde{W}_{k_{0}+1} & -I_{N-P} & \ldots & 0 \\
\cdot & \ldots & \ldots & \ldots & \cdot \\
\cdot & \ldots & \ldots & \ldots & \cdot \\
0 & \ldots & \ldots & \tilde{W}_{k_{1}-1} & -I_{N-P}
\end{array}\right]
$$

Let $\overline{\mathcal{J}}=\Pi_{1} \mathcal{J} \Pi_{2}$,

$$
\hat{\mathcal{J}}=\left[\begin{array}{cc}
\mathbf{I}_{\left(k_{1}-k_{0}\right) P} & P_{2} \\
0 & \tilde{\mathbf{C}} \\
0 & \tilde{\mathbf{W}}
\end{array}\right],
$$

and

$$
\tilde{\mathcal{J}}=\left[\begin{array}{c}
\tilde{\mathbf{C}} \\
\tilde{\mathbf{W}}
\end{array}\right] .
$$

Notice that $\overline{\mathcal{J}}$ is block lower triangular with the blocks over the diagonal $\mathbf{I}_{\left(k_{1}-k_{0}+1\right) P}$ and $\hat{\mathcal{J}}$. This implies $\operatorname{rank}(\overline{\mathcal{J}})=$ $\left(k_{1}-k_{0}+1\right) P+\operatorname{rank}(\hat{\mathcal{J}}) . \hat{\mathcal{J}}$ is block upper triangular with blocks over the diagonal $\mathbf{I}_{\left(k_{1}-k_{0}\right) P}$ and $\tilde{\mathcal{J}}$. Therefore, the following holds:

$\operatorname{rank}(\overline{\mathcal{J}})=\left(k_{1}-k_{0}+1\right) P+\left(k_{1}-k_{0}\right) P+\operatorname{rank}(\tilde{\mathcal{J}})$.

From Prop. 4 we know that $\left\{W_{k}, A_{B}, A_{C}\right\}_{k_{0}}^{k_{1}}$ is ISO over $\left[k_{0}, k_{1}\right]$ if and only if rank $(\overline{\mathcal{J}})=\left(k_{1}-k_{0}\right) P+\left(k_{1}-k_{0}+1\right) N$, which in turn is equivalent to $\operatorname{rank}(\tilde{\mathcal{J}})=\left(k_{1}-k_{0}+1\right)(N-$ $P)$. From Prop. 3, the latter corresponds to observability of $\left\{\tilde{W}_{k}, \tilde{A}_{C}\right\}_{k_{0}}^{k_{1}}$ over $\left[k_{0}, k_{1}\right]$.

The result in Prop. 5 can be interpreted as follows: when the subsystem $\left\{\tilde{W}_{k}, \tilde{A}_{C}\right\}_{k_{0}}^{k_{1}}$ is observable over $\left[k_{0}, k_{1}\right]$, one of the two unknown inputs in (3), namely $\tilde{x}_{k}$, is known and hence it is possible to compute $u_{k}$, since $\hat{x}_{k}$ is directly measured.

For LTI systems, alternatively, the PBH rank test may also be used to prove Prop. 5. The interested reader may be inspired by Prop. 4 in [20] and proof therein. 
As mentioned previously, Prop 1 and Prop. 4 characterize ISO in terms of rank conditions of matrices, namely, $\Psi_{k_{0}, k_{1}}$ and $\mathcal{J}_{k_{0}, k_{1}}$ respectively. These algebraic techniques work well provided we have access to the exact values of all the coefficients of the aforesaid matrices. Moreover, from a computational standpoint this technique is rather limited since the computational complexity increases as the size of the network grows. Therefore, in the sequel we turn our attention to structural (resp. s-structural) results, i.e., the focus is on finding conditions such that the system is ISO for almost all choices of free parameters (resp. every choice of free parameters) of the system matrices.

\section{Structural ISO}

The main objective of this section is to characterize ISO for almost all choices of free parameters.

\section{A. Definition and Implications}

We denote by $\left\{\mathcal{W}, A_{B}, A_{C}\right\}_{\text {LTV }}$ the family of all LTV systems as given in (1) and having the same zero/non-zero pattern as given by $\mathcal{W}, A_{B}$ and $A_{C}$ while $\left\{\mathcal{W}, A_{B}, A_{C}\right\}_{\text {LTI }}$ represents the corresponding family of all LTI systems.

As mentioned previously, structured systems have fixed zero positions and free parameters. Let $|\mathcal{E}|$ denote the number of ones in $A_{\mathcal{G}}$. Under such a setup, the free parameters can take values in $\mathbb{R}^{(|\mathcal{E}|)\left(k_{1}-k_{0}\right)}$, where $\left[k_{0}, k_{1}\right]$ represents the time window over which the system $\left\{\mathcal{W}, A_{B}, A_{C}\right\}_{\text {LTV }}$ is being observed. Notice that each element in $\mathbb{R}^{(|\mathcal{E}|)\left(k_{1}-k_{0}\right)}$ yields a choice of free parameters. Structural ISO then asks that there be at least one member in $\left\{\mathcal{W}, A_{B}, A_{C}\right\}_{\text {LTV }}$ which is observable. This leads us to the following definition.

Definition 4: $\left\{\mathcal{W}, A_{B}, A_{C}\right\}_{\mathrm{LTV}}$ is structurally ISO on $\left[k_{0}, k_{1}\right], k_{1}>k_{0}$ if there exists at least one system $\left\{W_{k}, A_{B}, A_{C}\right\}_{k_{0}}^{k_{1}}$ with $W_{k} \in \mathcal{W}$; such that $\left\{W_{k}, A_{B}, A_{C}\right\}_{k_{0}}^{k_{1}}$ is ISO.

Analogously, one can define structural observability and uniform $N$-step structural observability for LTV systems. In particular, definitions for structural ISO, structural observability and uniform $N$-step structural observability can also be obtained for LTI systems where the space of free parameters is $\mathbb{R}^{|\mathcal{E}|}$ and the same free parameters are repeated at each time instant.

It is well-known that observability (and controllability) is a property (see [29], [12]) such that either there is no choice of parameters that makes it true, or it is true for almost all choices of parameters. Almost all choices of parameters means all choices of parameter except those lying in some proper algebraic variety of the space of free parameters. This means there are some non-trivial polynomials (one or more, but finitely many) such that the property is true for all parameters except those which are zeros of this system of polynomials. The polynomials being non-trivial (i.e., not identically zero) ensures that the variety is proper (i.e., not the whole space of free parameters) and therefore, has Lebesgue measure zero. This can be interpreted as the property being true with probability one, if the parameters are thrown at random, according to any continuous probability distribution. Furthermore, small variations in the parameter values would not lead to loss of property. It turns out that the above discussion also holds for ISO, as shown in the following:

Proposition 6: The set of parameters for which $\left\{\mathcal{W}, A_{B}, A_{C}\right\}_{\text {LTV }}$ is not ISO is either the whole parameter space $\mathbb{R}^{(|\mathcal{E}|)\left(k_{1}-k_{0}\right)}$ or a proper variety of $\mathbb{R}^{(|\mathcal{E}|)\left(k_{1}-k_{0}\right)}$.

Proof: The proof is based on standard tools and is reported in Appendix.

This means that over a given interval $\left[k_{0}, k_{1}\right]$, if one member of the family of systems $\left\{\mathcal{W}, A_{B}, A_{C}\right\}_{\text {LTV }}$ is ISO then almost all members of $\left\{\mathcal{W}, A_{B}, A_{C}\right\}_{\text {LTV }}$ are ISO. On the other hand, if $\left\{\mathcal{W}, A_{B}, A_{C}\right\}_{\mathrm{LTV}}$ is not structurally ISO, then none of the members of $\left\{\mathcal{W}, A_{B}, A_{C}\right\}_{\text {LTV }}$ is ISO. An analogous result to Prop. 6 also holds for LTI systems wherein the space of free parameters $\mathbb{R}^{|\mathcal{E}|}$. Definition 4 needs to be seen against this backdrop.

It turns out that structural ISO for a family of LTI systems implies structural ISO for the corresponding family of LTV systems, and is given by the following remark

Remark 3: If the LTI system $\left\{\mathcal{W}, A_{B}, A_{C}\right\}_{\text {LTI }}$ is structurally ISO, then the corresponding LTV system $\left\{\mathcal{W}, A_{B}, A_{C}\right\}_{\text {LTV }}$ is structurally ISO over all sufficiently long intervals. Indeed, if the system $\left\{\mathcal{W}, A_{B}, A_{C}\right\}_{\text {LTI }}$ is structurally ISO, then there exists $W \in \mathcal{W}$, such that the triplet $\left(W, A_{B}, A_{C}\right)$ is ISO. Therefore, over an interval $\left[k_{0}, k_{1}\right]$ of length at least $N$, one can set $W_{k}=W, \forall k \in\left[k_{0}, k_{1}\right]$, obtaining a system $\left\{W_{k}, A_{B}, A_{C}\right\}_{k_{0}}^{k_{1}}$ that is ISO over $\left[k_{0}, k_{1}\right]$, thereby exhibiting a choice of entries for which $\left\{\mathcal{W}, A_{B}, A_{C}\right\}_{\text {LTV }}$ is ISO. Consequently, from Definition 4 , the system $\left\{\mathcal{W}, A_{B}, A_{C}\right\}_{\text {LTV }}$ is structurally ISO over $\left[k_{0}, k_{1}\right]$.

However, the converse of Remark 3 is open. In the rest of this section we show that, under assumption A2, the conditions given in Remark 3 are equivalent.

\section{B. Uniform N-step structural ISO for LTV systems}

From Proposition 5, we can study ISO by studying observability of a suitable sub-system. Here we apply this technique to the family of systems $\left\{\mathcal{W}, A_{B}, A_{C}\right\}_{\text {LTV }}$, defining a suitable family of subsystems. We define the set of matrices $\tilde{\mathcal{W}}$ as $\tilde{\mathcal{W}}=\left\{\bar{Q}_{N}^{T} W \bar{Q}_{N} \mid W \in \mathcal{W}\right\}$. Let $\left\{\tilde{\mathcal{W}}, \tilde{A}_{C}\right\}_{\text {LTV }}$ represent the family of all LTV systems as given in (4) but without the known input $\hat{x}_{k}$. We denote by $\left\{\tilde{\mathcal{W}}, \tilde{A}_{C}\right\}_{\text {LTI }}$ the counterpart LTI subsystem (i.e., whose matrices have the same zero/nonzero pattern as given by $\tilde{\mathcal{W}}$ ). As a consequence of Prop. 5, for LTI systems we have the following remark:

Remark 4: Under A2, $\left\{\mathcal{W}, A_{B}, A_{C}\right\}_{\text {LTI }}$ is uniform $N$ step structural ISO if and only if $\left\{\tilde{\mathcal{W}}, \tilde{A}_{C}\right\}_{\text {LTI }}$ is structurally observable.

It turns out that corresponding to Remark 4, conditions for structural results can also be obtained for LTV systems, as shall be evidenced in the rest of this subsection.

An immediate corollary of Prop. 5 is the following

Proposition 7: Under A2, $\left\{\mathcal{W}, A_{B}, A_{C}\right\}_{\text {LTV }}$ is structurally ISO over $\left[k_{0}, k_{1}\right]$ if and only if $\left\{\tilde{\mathcal{W}}, \tilde{A}_{C}\right\}_{\text {LTV }}$ is structurally observable over $\left[k_{0}, k_{1}\right]$.

The advantage of Prop. 7 is that it breaks down the problem of structural ISO into an equivalent problem in structural observability. With this in hand, and rewriting Thm. 3 in [30] 
(also see [16]) for observability we obtain equivalence between structural observability for LTV and LTI systems, and is given by the following proposition.

Proposition 8 (Thm. 3 in [30]): Under A2, over any interval $\left[k_{0}, k_{1}\right]$ of length at least $N,\left\{\tilde{\mathcal{W}}, \tilde{A}_{C}\right\}_{\text {LTV }}$ is structurally observable if and only if $\left\{\tilde{\mathcal{W}}, \tilde{A}_{C}\right\}_{\text {LTI }}$ is structurally observable.

Prop. 7 and Prop. 8 together break down the structural ISO problem of LTV systems into a structural observability problem of a corresponding suitably defined LTI subsystem. Thanks to [21] it turns out that the structural observability of an LTI subsystem can be determined by checking certain graph-theoretical conditions. Before proceeding, we need a few constructs on $\mathcal{G}$. Let $\tilde{\mathcal{G}}$ be the graph corresponding to $\tilde{\mathcal{W}}$. Let $\mathcal{S}=\left\{\mathcal{L}_{1}, \mathcal{L}_{2}, \mathcal{E}_{S}\right\}$ be a bipartite graph associated with $\tilde{\mathcal{G}}$, with $\mathcal{L}_{1}=\tilde{\mathcal{V}} \backslash \tilde{\mathcal{O}}, \mathcal{L}_{2}=\tilde{\mathcal{V}}$ constructed in the following manner, two vertices in $\mathcal{L}_{1}$ and $\mathcal{L}_{2}$ that correspond to the same element $v \in \tilde{\mathcal{V}}$ are denoted as $u_{v}$ and $w_{v}$ respectively, and $\mathcal{E}_{S}=\left\{\left(u_{i}, w_{j}\right) \in \mathcal{L}_{1} \times \mathcal{L}_{2} \mid(i, j) \in \tilde{\mathcal{E}}\right\}$. Similar to [31] but without the introduction of additional output nodes in $\tilde{\mathcal{G}}$ we state the following definitions.

Definition 5: The graph $\tilde{\mathcal{G}}$ with observation set $\tilde{\mathcal{O}}$ is said to be output-connected if for all $v \in \tilde{V}$, there exists a path from $v$ to $w$ for some $w \in \tilde{\mathcal{O}}$.

Definition 6: A matching is a set of edges that do not share any common vertices.

With Definitions 5 and 6 in hand, we state the following result, rephrased for observability

Lemma 1 (Thm. 1 [14]): The system $\left\{\tilde{\mathcal{W}}, \tilde{A}_{C}\right\}_{\text {LTI }}$ is structurally observable if and only if:

1) $\tilde{\mathcal{G}}$ is output-connected;

2) there exists a matching in $\mathcal{S}$ of size $N-|\tilde{\mathcal{O}}|$.

As an aside, the above result previously appeared in [21] and [31]. With Lemma 1 in place we state our first main result.

Theorem 1: Under A2, $\left\{\mathcal{W}, A_{B}, A_{C}\right\}_{\mathrm{LTV}}$ is uniformly $N$ step structurally ISO if and only if the following conditions are satisfied:

1) $\tilde{\mathcal{G}}$ is output-connected;

2) there exists a matching in $\mathcal{S}$ of size $N-|\tilde{\mathcal{O}}|$.

Proof: From Prop. 7, it can be seen that under A2, the system $\left\{\mathcal{W}, A_{B}, A_{C}\right\}_{\text {LTV }}$ is structurally ISO over $\left[k_{0}, k_{1}\right]$ if and only if the subsystem $\left\{\tilde{\mathcal{W}}, \tilde{A}_{C}\right\}_{\text {LTV }}$ is structurally observable over $\left[k_{0}, k_{1}\right]$, while from Prop. 8 it can be seen that the subsystem $\left\{\tilde{\mathcal{W}}, \tilde{A}_{C}\right\}_{\text {LTV }}$ is structurally observable over $\left[k_{0}, k_{1}\right]$ if and only if the corresponding LTI subsystem $\left\{\tilde{\mathcal{W}}, \tilde{A}_{C}\right\}_{\text {LTI }}$ is structurally observable. It is well-known that LTI systems are either observable over every sufficiently long interval or not observable at all. Thus, setting $\delta=N$ in Remark 1, and from Prop. 7 and Prop. 8, it follows that under A2, the system $\left\{\mathcal{W}, A_{B}, A_{C}\right\}_{\text {LTV }}$ is structurally ISO over $\left[k_{0}, k_{1}\right]$ if and only if the subsystem $\left\{\tilde{\mathcal{W}}, \tilde{A}_{C}\right\}_{\text {LTI }}$ is uniform N-step structural ISO. Thereafter, from Lemma 1 , the proof is complete.

Example 1: With reference to the system shown in Figure 1, it can be seen from Figure 2 and Figure 4 that the subsystem is output-connected and its bipartite graph $\mathcal{S}$ contains a matching of size $N-|\tilde{\mathcal{O}}|$ and hence, the subsystem is structurally observable [14]. Therefore, from Thm 1, the system given in

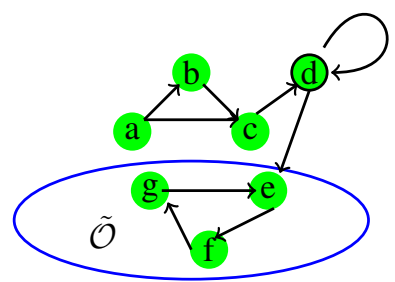

Fig. 2: The subsystem $\tilde{\mathcal{G}}$ for the system shown in Fig. 1

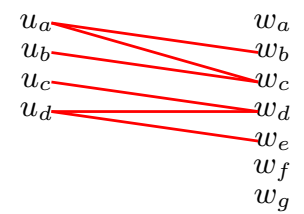

Fig. 3: Bipartite graph $\mathcal{S}$ associated with $\tilde{\mathcal{G}}$

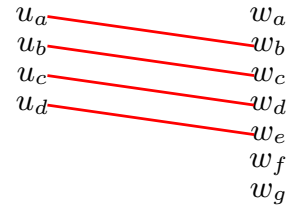

Fig. 4: A Matching $\mathcal{M}$ in $\mathcal{S}$

Figure 1 is uniformly $N$-step structurally ISO

Item i) or output-connectedness of $\tilde{\mathcal{G}}$ can be checked by using a variant of Tarjan's algorithm and has complexity that is linear in the number of edges and vertices of $\tilde{\mathcal{G}}$ (i.e., $O(|\tilde{\mathcal{E}}|+|\tilde{\mathcal{V}}|)$ ) [32]. On the other hand, Hopcroft-Karp maximum matching algorithm can be used for checking item ii) and its complexity is $O((|\tilde{\mathcal{E}}|+|\tilde{\mathcal{V}}|) \sqrt{|\tilde{\mathcal{V}}|})[33]$.

\section{S-STRUCTURAL ISO}

The main objective of this section is to characterize ISO for every choice of entry in $\mathcal{W}^{*}$.

\section{A. Definition}

S-structural properties are those that hold for every non-zero choice of free parameters of the system matrices. That is, sstructural ISO (resp. observability) requires that every member of the family of LTV systems given by $\left\{\mathcal{W}^{*}, A_{B}, A_{C}\right\}_{\mathrm{LTV}}$, be ISO (resp. observable). This leads us to the following definition.

Definition 7: Let $k_{1}, k_{0} \in \mathbb{Z}$ and $k_{1}>k_{0}$, $\left\{\mathcal{W}^{*}, A_{B}, A_{C}\right\}_{\text {LTV }} \quad$ is s-structurally ISO on $\left[k_{0}, k_{1}\right]$ if for every system $\left\{W_{k}, A_{B}, A_{C}\right\}_{k_{0}}^{k_{1}}$ with $W_{k} \in \mathcal{W}^{*}$, $\left\{W_{k}, A_{B}, A_{C}\right\}_{k_{0}}^{k_{1}}$ is ISO.

Analogous to Definition 7, one can also define s-structural observability and uniform N-step s-structural observability for LTV systems. In particular, definitions for $s$-structural ISO, s-structural observability and uniform $N$-step s-structural observability can also be obtained for LTI systems.

It turns out that s-structural ISO for a family of LTV systems 
implies s-structural ISO for the corresponding family of LTI systems, and is given by the following remark

Remark 5: If the system $\left\{\mathcal{W}^{*}, A_{B}, A_{C}\right\}_{\text {LTV }}$ is s-structurally ISO over an interval $\left[k_{0}, k_{1}\right]$, then the corresponding LTI system $\left\{\mathcal{W}^{*}, A_{B}, A_{C}\right\}_{\text {LTI }}$ is s-structurally ISO. Indeed, if the system $\left\{\mathcal{W}^{*}, A_{B}, A_{C}\right\}_{\text {LTI }}$ is not s-structurally ISO, then from Definition 7 there exists a system $\left\{W_{1}, A_{B}, A_{C}\right\}$ with $W_{1} \in \mathcal{W}^{*}$ such that $\left\{W_{1}, A_{B}, A_{C}\right\}$ is not ISO. Over any interval $\left[k_{0}, k_{1}\right]$, we can set $W_{k}=W_{1} \forall k \in\left[k_{0}, k_{1}\right]$ such that the LTV system $\left\{W_{k}, A_{B}, A_{C}\right\}_{k_{0}}^{k_{1}}$ is not ISO. Consequently, from Definition 7, the system $\left\{\mathcal{W}^{*}, A_{B}, A_{C}\right\}_{\mathrm{LTV}}$ is not sstructurally ISO over any interval.

Notice that for structural ISO the implication is in the other direction (see Remark 3).

The converse of Remark 5 remains open. In the rest of this section we show that, under assumption A2, over sufficiently long intervals, the conditions given in Remark 5 are equivalent.

\section{B. Uniform N-step s-structural ISO for LTV systems}

The set of matrices $\tilde{\mathcal{W}}^{*}$ is defined analogous to $\tilde{\mathcal{W}}$. One can use Prop. 5 so as to obtain s-structural ISO results. We first focus on LTI systems. As another consequence of Prop. 5, we have the following:

Remark 6: Under A2, $\left\{\mathcal{W}, A_{B}, A_{C}\right\}_{\text {LTI }}$ is uniform $N$-step s-structural ISO if and only if $\left\{\tilde{\mathcal{W}}, \tilde{A}_{C}\right\}_{\text {LTI }}$ is s-structurally observable.

It turns out that one can obtain similar conditions for LTV systems as well, as shall be seen in the rest of this subsection. First notice that another immediate corollary of Prop. 5 can be stated as follows

Proposition 9: Under A2, $\left\{\mathcal{W}^{*}, A_{B}, A_{C}\right\}_{\mathrm{LTV}} \quad$ is $\mathrm{s}-$ structurally ISO over $\left[k_{0}, k_{1}\right]$ if and only if $\left\{\tilde{\mathcal{W}}^{*}, \tilde{A}_{C}\right\}_{\text {LTV }}$ is s-structurally observable over $\left[k_{0}, k_{1}\right]$.

Thanks to Prop. 9, we can now rephrase s-structural ISO of a family of LTV systems as an equivalent problem in s-structural observability of a suitable family of LTV systems. Against this backdrop, it is indeed relevant to see if s-structural ISO of LTV systems is equivalent to s-structural observability of a suitable family of LTI systems. The following proposition is immediate from Corollary IV.2 [17].

Proposition 10: Under A2, over any interval $\left[k_{0}, k_{1}\right]$ of length at least $N,\left\{\tilde{\mathcal{W}}^{*}, \tilde{A}_{C}\right\}_{\text {LTV }}$ is s-structurally observable if and only if $\left\{\tilde{\mathcal{W}}^{*}, \tilde{A}_{C}\right\}_{\text {LTI }}$ is s-structurally observable.

Thus, from Prop. 9 and Prop. 10, it can be seen that under assumption A2, the s-structural ISO problem for LTV systems breaks down into an equivalent problem in s-structural observability for a suitably defined LTI subsystem. This equivalence allows us to exploit the literature on s-structural observability, as we see in the following.

Thanks to [14], (also see [15]), it turns out that s-structural observability of an LTI system can be assessed by checking some graph-theoretical conditions. Here we would be focusing on the notion of uniquely restricted matching (also known as constrained matching) as in [14]. In order to proceed, a few constructs on the graph $\tilde{\mathcal{G}}$ are due. $\mathcal{E}_{\text {loop }} \subset \mathcal{E}_{\mathcal{S}}$ denote the edges of the form $\left\{u_{i}, w_{i}\right\}$ if there exists any. Notice that $\mathcal{E}_{\text {loop }}$ corresponds to self-loops in $\tilde{\mathcal{G}}$. Let $\mathcal{E}_{\text {new }}$ denote the set of newly added self-loops in $\tilde{\mathcal{G}}$ i.e., adding self-loops for those vertices $i \in \tilde{\mathcal{V}}$ that previously did not have one in $\tilde{\mathcal{G}}$. Let $\mathcal{S}_{\times}=\left\{\mathcal{L}_{1}, \mathcal{L}_{2}, \mathcal{E}_{\mathcal{S}_{\times}}\right\}$, where $\mathcal{E}_{\mathcal{S}_{\times}}=\left\{\mathcal{E}_{\mathcal{S}} \cup \mathcal{E}_{\text {new }}\right\}$, denote another bipartite graph on $\tilde{\mathcal{G}}$. We recall that a matching is said to be uniquely restricted if there is no other matching involving the same vertex set. Equivalent characterizations of uniquely restricted matchings are discussed in [34]. The following result is the same as Thm. 5 in [14] but rewritten for s-structural observability.

Lemma 2 (Thm. 5 [14]): The system $\left\{\tilde{\mathcal{W}}^{*}, \tilde{A}_{C}\right\}_{\text {LTI }}$ is sstructurally observable if and only if:

1) there exists a uniquely restricted matching $\mathcal{M} \subseteq \mathcal{E}_{\mathcal{S}}$ of size $N-|\tilde{\mathcal{O}}|$

2) there exists a uniquely restricted matching $\mathcal{M}_{\times} \subseteq \mathcal{E}_{\mathcal{S}_{\times}}$ of size $N-|\tilde{\mathcal{O}}|$ such that $\mathcal{M}_{\times} \cap \mathcal{E}_{\text {loop }}=\emptyset$.

With Lemma 2 in place we present our second main result in the following theorem.

Theorem 2: Under A2, $\left\{\mathcal{W}^{*}, A_{B}, A_{C}\right\}_{\mathrm{LTV}}$ is uniformly $N$-step s-structurally ISO if and only if the following two conditions are satisfied:

1) there exists a uniquely restricted matching $\mathcal{M} \subseteq \mathcal{E}_{\mathcal{S}}$ of size $N-|\tilde{\mathcal{O}}|$

2) there exists a uniquely restricted matching $\mathcal{M}_{\times} \subseteq \mathcal{E}_{\mathcal{S}_{\times}}$ of size $N-|\tilde{\mathcal{O}}|$ such that $\mathcal{M}_{\times} \cap \mathcal{E}_{\text {loop }}=\emptyset$.

Proof: From Prop. 9 and Prop. 10, it can be seen that under A2, $\left\{\mathcal{W}^{*}, A_{B}, A_{C}\right\}_{\text {LTV }}$ is s-structurally ISO over any interval of length at least $N$ if and only if $\left\{\tilde{\mathcal{W}}^{*}, \tilde{A}_{C}\right\}_{\text {LTI }}$ is s-structurally observable, while from Prop. 10, it can also be seen that $\left\{\tilde{\mathcal{W}}^{*}, \tilde{A}_{C}\right\}_{\text {LTI }}$ is s-structurally observable if and only if $\left\{\tilde{\mathcal{W}}^{*}, \tilde{A}_{C}\right\}_{\mathrm{LTV}}$ is s-structurally observable over every interval of length at least $N$. Thus, Prop. 9 and Prop. 10 together with setting $\delta=N$ in Remark 1, results in the following: under $\mathrm{A} 2,\left\{\mathcal{W}^{*}, A_{B}, A_{C}\right\}_{\mathrm{LTV}}$ is s-structurally ISO if and only if the LTI subsystem $\left\{\tilde{\mathcal{W}}^{*}, \tilde{A}_{C}\right\}_{\text {LTI }}$ is uniform $N$ step s-structural ISO. Thereafter, from Lemma 2, the proof is complete.

The conditions in Theorem 2 can be checked using the algorithm given in [14], with complexity $O\left(|\tilde{\mathcal{V}}|^{2}\right)$, or with the algorithm introduced in [35], which achives a linear complexity $O(|\tilde{\mathcal{V}}|+|\tilde{\mathcal{E}}|)$ by combining sophisticated data structures and sparse matrix techniques.

Example 1 (continued): With respect to the system given in Figure 1, first recall that $\mathcal{M}=$ $\left\{\left(u_{a}, w_{b}\right),\left(u_{b}, w_{c}\right),\left(u_{c}, w_{d}\right),\left(u_{d}, w_{e}\right)\right\}$ (see Figure 4) is a matching in $\mathcal{S}$. Furthermore, there exists no other matching $\tilde{\mathcal{M}} \subset \mathcal{E}_{\mathcal{S}}$ saturating the same vertices as $\mathcal{M}$, and hence, by definition $\mathcal{M} \subset \mathcal{E}_{S}$ is a uniquely restricted matching. The second condition is checked with respect to the bipartite graph $\mathcal{S}_{\times}$given in Figure 5 . It can be seen that $\mathcal{M}_{\times}=\left\{\left(u_{a}, w_{a}\right),\left(u_{b}, w_{b}\right),\left(u_{c}, w_{c}\right),\left(u_{d}, w_{e}\right)\right\}$ (see Figure 6) is a matching in $\mathcal{S}_{\times}$that satisfies $\mathcal{M}_{\times} \cap \mathcal{E}_{\text {loop }}=\emptyset$. Notice that there exists no other matching $\hat{\mathcal{M}}_{\times} \subset \mathcal{E}_{\mathcal{S}_{\times}}$saturating the same vertices as $\mathcal{M}_{\times}$. Therefore, from Thm. 2, the system given in Figure 1 is uniformly $N$-step s-structurally ISO. Now consider another example. 


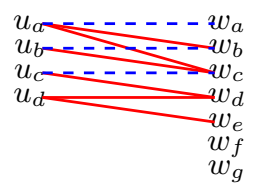

Fig. 5: Bipartite graph $\mathcal{S}_{\times}$, in solid red: $\mathcal{S}_{\times} \cap \mathcal{S}$, in dashed blue: $\mathcal{E}_{\text {new }}=\left\{\left(u_{a}, w_{a}\right),\left(u_{b}, w_{b}\right),\left(u_{c}, w_{c}\right)\right\}$ while $\mathcal{E}_{\text {loop }}=$ $\left\{\left(u_{d}, w_{d}\right)\right\}$

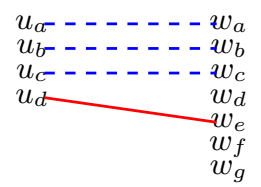

Fig. 6: A matching $\mathcal{M}_{\times}$in $\mathcal{S}_{\times}$

Example 2: Consider the system given in Figure 7, whose corresponding subsystem is given in Figure 8, while a bipartite graph associated with the subsystem $\tilde{\mathcal{G}}_{1}$ is given in Figure 9. It is immediate that the subsystem $\tilde{\mathcal{G}}_{1}$ is output-connected. Furthermore, there also exists a matching of size $N-|\tilde{\mathcal{O}}|$ on the bipartite graph $\mathcal{S}_{1}$. Therefore from Thm. 1 the system given in Figure 7 is uniformly $N$-step structurally ISO. On the other hand, from Figures 10(a) and 10(b) it can be seen that there does not exist a uniquely restricted matching over the choice of vertex sets $\left\{u_{a}, u_{b}, u_{c}\right\}$ and $\left\{w_{a}, w_{b}, w_{c}\right\}$. The same can be said with respect to the vertex sets $\left\{u_{a}, u_{b}, u_{c}\right\}$ and $\left\{w_{a}, w_{b}, w_{d}\right\}$ (see Figures 10(c) and 10(d)), $\left\{u_{a}, u_{b}, u_{c}\right\}$ and $\left\{w_{b}, w_{c}, w_{d}\right\}$ (see Figures 10(e) and 10(f)), $\left\{u_{a}, u_{b}, u_{c}\right\}$ and $\left\{w_{a}, w_{c}, w_{d}\right\}$ (see Figures $10(\mathrm{~g})$ and 10(h)). Thus, there does not exist a uniquely restricted matching of size $N-|\tilde{\mathcal{O}}|$ on the bipartite graph $\mathcal{S}_{1}$, and hence, from Thm. 2, the system given in Figure 7 is not uniformly $N$-step s-structurally ISO.

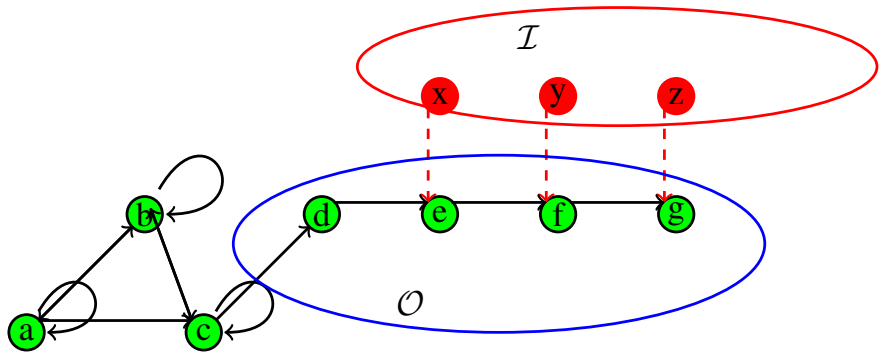

Fig. 7: The system $\mathcal{G}_{1}$

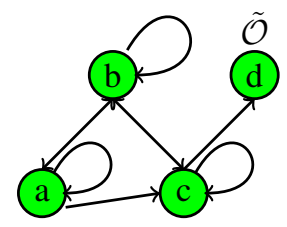

Fig. 8: Subsystem $\tilde{\mathcal{G}}_{1}$
Fig. 9: Bipartite graph $\mathcal{S}_{1}$ associated with $\tilde{\mathcal{G}}_{1}$

\section{CONCLUSION}

We have studied ISO with one time-step delay for linear network systems with a fixed topology. Under the assumptions: (i) each assailable node can be attacked by at most a single unknown input, (ii) each unknown input affects exactly one node, and (iii) direct measurements of certain states are available, we provide a characterization of ISO in terms of observability of a suitably defined subsystem. Moreover, we have studied uniform $N$-step ISO for almost all choices of free parameters in the $\mathcal{W}$ as well as for all non-zero choices of free parameters in $\mathcal{W}$, where $\mathcal{W}$ stands for the family of systems sharing the same zero/non-zero pattern. A future line of investigation would be to characterize ISO for more general linear network systems by considering time-varying topology and less a priori knowledge on the assailable nodes. A weaker notion of ISO, wherein the focus is on the unique recovery of just a subset of the states, for instance, only the states that are not affected by an unknown input, could be another direction of future works.

\section{APPENDIX}

\section{Proof of Proposition 2}

Item i) requires that the first $N$ columns of $\Psi_{k_{0}, k_{1}}$ be linearly independent. Item ii) requires that the last $P$ columns of $\Psi_{k_{0}, k_{1}}$ be linearly independent, while items iii) and iv) are necessary conditions for item ii). To see the necessity of items v) and vi), notice that, in order for $\Psi_{k_{0}, k_{1}}$ to be full column rank, it is necessary that $\Psi_{k_{0}, k_{1}}$ has at least as many rows as columns, i.e.,

$$
M\left(k_{1}-k_{0}+1\right) \geq N+\left(k_{1}-k_{0}\right) P .
$$

From the above equation, since $\left(k_{1}-k_{0}+1\right)>0$, it follows that $M \geq P+\frac{N-P}{\left(k_{1}-k_{0}+1\right)}$. If $N>P$, this implies that $M>P$. Then, under $M>P$, item vi) immediately follows from the above equation.

For the particular case of $M=P=N$, notice that $\Psi_{k_{0}, k_{1}}$ is a block lower triangular matrix with each of the blocks being square. Hence, a necessary and sufficient condition for full column rank of $\Psi_{k_{0}, k_{1}}$ is that each of the diagonal blocks have full column rank. This is equivalent to, i) $\operatorname{rank}\left(A_{C} A_{B}\right)=N$ $\forall k \in\left[k_{0}+1, k_{1}\right]$ and ii) $\operatorname{rank}\left(A_{C}\right)=N$. Notice that $\forall k \in$ $\left[k_{0}+1, k_{1}\right], \operatorname{rank}\left(A_{C} A_{B}\right)=N$ if and only if: i) $\operatorname{rank}\left(A_{C}\right)=$ $N$ and ii) $\operatorname{rank}\left(A_{B}\right)=N$.

\section{Proof of Proposition 6}

From Prop. 1, ISO is equivalent to $\Psi_{k_{0}, k_{1}}$ having rank $N+$ $\left(k_{1}-k_{0}\right) P$. Hence, the system is not ISO if and only if all the square submatrices of size $N+\left(k_{1}-k_{0}\right) P$ have zero determinant. Notice that the entries of $\Psi_{k_{0}, k_{1}}$ are polynomials, whose variables are the free parameters of $W_{k}, A_{B}$ and $A_{C}$; the fixed values (zeros) can be interpreted as polynomials of degree zero. For each submatrix of size $N+\left(k_{1}-k_{0}\right) P$, the determinant is obtained by multiplications and summations of such polynomials, and hence is itself a polynomial. We have found a finite set of polynomials, such that the system is not ISO if and only if the parameters belong to the zero set of all 


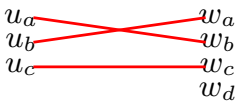

(a) Matching $\mathcal{M}_{1}$ on $\mathcal{S}_{1}$

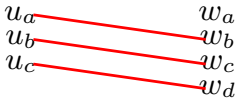

(e) Matching $\mathcal{M}_{5}$ on $\mathcal{S}_{1}$

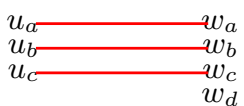

(b) Matching $\mathcal{M}_{2}$ on $\mathcal{S}_{1}$

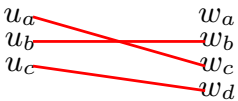

(f) Matching $\mathcal{M}_{6}$ on $\mathcal{S}_{1}$

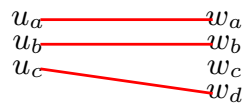

(c) Matching $\mathcal{M}_{3}$ on $\mathcal{S}_{1}$

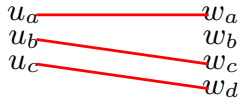

(g) Matching $\mathcal{M}_{7}$ on $\mathcal{S}_{1}$

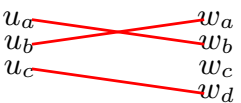

(d) Matching $\mathcal{M}_{4}$ on $\mathcal{S}_{1}$

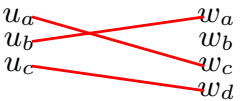

(h) Matching $\mathcal{M}_{8}$ on $\mathcal{S}_{1}$

Fig. 10: All maximum matchings on $\mathcal{S}_{1}$

these polynomials. Either such polynomials are all trivial (i.e., constantly equal to zero) and hence all choices of parameters result in a system not ISO, or at least one of the polynomials is non-trivial, and hence the set of parameters for which the system is not ISO is a proper variety of parameter space.

\section{REFERENCES}

[1] F. Pasqualetti, F. Dorfler, and F. Bullo, "Control-theoretic methods for cyberphysical security: Geometric principles for optimal cross-layer resilient control systems," IEEE Control Systems, vol. 35, no. 1, pp. 110-127, 2015.

[2] J. Slay and M. Miller, "Lessons learned from the Maroochy water breach," in International Conference on Critical Infrastructure Protection. Springer, 2007, pp. 73-82.

[3] J. P. Conti, "The day the samba stopped," Engineering \& Technology, vol. 5, no. 4, pp. 46-47, 2010

[4] M. Hautus, "Strong detectability and observers," Linear Algebra and its applications, vol. 50, pp. 353-368, 1983.

[5] P. K. Kitanidis, "Unbiased minimum-variance linear state estimation," Automatica, vol. 23, no. 6, pp. 775-778, 1987.

[6] C.-S. Hsieh, "Robust two-stage Kalman filters for systems with unknown inputs," IEEE Transactions on Automatic Control, vol. 45, no. 12, pp. $2374-2378,2000$

[7] S. Gillijns and B. De Moor, "Unbiased minimum-variance input and state estimation for linear discrete-time systems," Automatica, vol. 43, no. 1, pp. 111-116, 2007.

[8] S. Z. Yong, M. Zhu, and E. Frazzoli, "A unified filter for simultaneous input and state estimation of linear discrete-time stochastic systems," Automatica, vol. 63, pp. 321-329, 2016.

[9] H. L. Trentelman, A. A. Stoorvogel, and M. Hautus, "Control theory for linear systems," Communications and Control Engineering Series, Springer, 2002.

[10] C.-T. Lin, "Structural controllability," IEEE Transactions on Automatic Control, vol. 19, no. 3, pp. 201-208, 1974.

[11] H. Mayeda and T. Yamada, "Strong structural controllability," SIAM Journal on Control and Optimization, vol. 17, no. 1, pp. 123-138, 1979.

[12] J.-M. Dion, C. Commault, and J. Van Der Woude, "Generic properties and control of linear structured systems: a survey," Automatica, vol. 39, no. 7, pp. 1125-1144, 2003.

[13] J. C. Jarczyk, F. Svaricek, and B. Alt, "Strong structural controllability of linear systems revisited," in Decision and Control and European Control Conference (CDC-ECC), 2011 50th IEEE Conference on. IEEE, 2011, pp. 1213-1218.

[14] A. Chapman and M. Mesbahi, "On strong structural controllability of networked systems: a constrained matching approach," in 2013 American Control Conference, Washington, DC, USA, 2013, pp. 61266131.

[15] M. Trefois and J.-C. Delvenne, "Zero forcing number, constrained matchings and strong structural controllability," Linear Algebra and its Applications, vol. 484, pp. 199-218, 2015.

[16] S. Poljak, "On the generic dimension of controllable subspaces," IEEE Transactions on Automatic Control, vol. 35, no. 3, pp. 367-369, 1990.

[17] G. Reissig, C. Hartung, and F. Svaricek, "Strong structural controllability and observability of linear time-varying systems," IEEE Transactions on Automatic Control, vol. 59, no. 11, pp. 3087-3092, 2014.

[18] T. Boukhobza, F. Hamelin, and S. Martinez-Martinez, "State and input observability for structured linear systems: A graph-theoretic approach," Automatica, vol. 43, no. 7, pp. 1204-1210, 2007.
[19] S. Gracy, F. Garin, and A. Y. Kibangou, "Strong structural input and state observability of LTV network systems with multiple unknown inputs," in 20th IFAC World Congress, Toulouse, France, 2017, pp. 7618-7623.

[20] A. Y. Kibangou, F. Garin, and S. Gracy, "Input and state observability of network systems with a single unknown input," ser. Proc. 6th IFAC Workshop on Distributed Estimation and Control in Networked Systems (NecSys). Tokyo, Japan: IFAC, Sep. 2016.

[21] C. Commault, J.-M. Dion, and J. W. van der Woude, "Characterization of generic properties of linear structured systems for efficient computations," Kybernetika, vol. 38, no. 5, pp. 503-520, 2002.

[22] A. Teixeira, H. Sandberg, and K. H. Johansson, "Networked control systems under cyber attacks with applications to power networks," in Proceedings of the 2010 American Control Conference, Baltimore, MD, USA, 2010, pp. 3690-3696.

[23] Y. Liu, P. Ning, and M. K. Reiter, "False data injection attacks against state estimation in electric power grids," ACM Transactions on Information and System Security, vol. 14, no. 1, p. 13, 2011.

[24] R. J. Patton, P. M. Frank, and R. N. Clarke, Fault diagnosis in dynamic systems: theory and application. Prentice-Hall, Inc., 1989.

[25] G. De Nicolao, G. Sparacino, and C. Cobelli, "Nonparametric input estimation in physiological systems: problems, methods, and case studies," Automatica, vol. 33, no. 5, pp. 851-870, 1997.

[26] R. Kalman, "On the general theory of control systems," IRE Transactions on Automatic Control, vol. 4, no. 3, pp. 110-110, 1959.

[27] W. S. Levine, The Control Handbook. CRC press, 1996.

[28] K. Murota, Matrices and matroids for systems analysis. Springer, 2000, vol. 20.

[29] R. W. Shields and J. B. Pearson, "Structural controllability of multi-input linear systems," Rice University ECE Technical Report, no. TR7502, 1975.

[30] S. Poljak, "On the gap between the structural controllability of timevarying and time-invariant systems," IEEE Transactions on Automatic Control, vol. 37, no. 12, pp. 1961-1965, 1992.

[31] C. Commault, J.-M. Dion, and D. H. Trinh, "Observability preservation under sensor failure," IEEE Transactions on Automatic Control, vol. 53, no. 6, pp. 1554-1559, 2008.

[32] L. Úbeda, C. Herrera, I. Barriales, P. J. Zufiria, and M. Congosto, "A combined algorithm for analyzing structural controllability and observability of complex networks," in Proceedings of the International Conference on Scientific Computing (CSC). The Steering Committee of The World Congress in Computer Science, Computer Engineering and Applied Computing (WorldComp), 2013, p. 112.

[33] J. E. Hopcroft and R. M. Karp, "A $\mathrm{n}^{5 / 2}$ algorithm for maximum matchings in bipartite graphs," in 12th Annual Symposium on Switching and Automata Theory, 1971. IEEE, 1971, pp. 122-125.

[34] M. C. Golumbic, T. Hirst, and M. Lewenstein, "Uniquely restricted matchings," Algorithmica, vol. 31, no. 2, pp. 139-154, 2001.

[35] A. Weber, G. Reissig, and F. Svaricek, "A linear time algorithm to verify strong structural controllability," in Decision and Control (CDC), 2014 IEEE 53rd Annual Conference on. IEEE, 2014, pp. 5574-5580. 